\title{
Intra-op Measurement of the Mechanical Axis Deviation: An Evaluation Study on 19 Human Cadaver Legs
}

\author{
Lejing Wang ${ }^{1}$, Pascal Fallavollita ${ }^{1}$, Alexander Brand ${ }^{2}$, Okan Erat ${ }^{1}$, \\ Simon Weidert ${ }^{2}$, Peter-Helmut Thaller ${ }^{2}$, Ekkehard Euler ${ }^{2}$, and Nassir Navab ${ }^{1}$ \\ ${ }^{1}$ Chair for Computer Aided Medical Procedures (CAMP), TU Munich, Germany \\ 2 Trauma Surgery Department, Klinikum Innenstadt, LMU Munich, Germany
}

\begin{abstract}
The alignment of the lower limb in high tibial osteotomy (HTO) or total knee arthroplasty (TKA) must be determined intraoperatively. One way to do so is to deform the mechanical axis deviation (MAD), for which a tolerance measurement of $10 \mathrm{~mm}$ is widely accepted. Many techniques are proposed in clinical practice such as visual inspection, cable method, grid with lead impregnated reference lines, or more recently, navigation systems. Each has their disadvantages including reliability of the MAD measurement, excess radiation, prolonged operation time, complicated setup and high cost. To alleviate such shortcomings, we propose a novel clinical protocol that allows quick and accurate intraoperative calculation of MAD. This is achieved by an X-ray stitching method requiring only three $\mathrm{X}$-ray images placed into a panoramic image frame during the entire procedure. The method has been systematically analyzed in a simulation framework in order to investigate its accuracy and robustness. Furthermore, we validated our protocol via a preclinical study comprising 19 human cadaver legs. Four surgeons determined MAD measurements using our X-ray panorama and compared these values to a gold-standard CT-based technique. The maximum average MAD error was $3.5 \mathrm{~mm}$ which shows great potential for the technique.
\end{abstract}

\section{Introduction and Related Work}

Patients with a condition known as knee osteoarthritis experience unfavorable wear and tear on the menisci and articular cartilage. These degenerative processes can cause the knee's protective tissues to wear on one side more than the other in a repetitive cycle of damage. A partial or total knee replacement can correct this condition when joint damage is beyond repair. A procedure known as a high tibial osteotomy (HTO), wedges open the upper tibia to reconfigure the knee joint. The weight-bearing part of the knee is shifted from degenerative or worn tissue onto healthier tissue. This procedure is typically reserved for younger patients with pain resulting from instability and malalignment. A high tibial osteotomy is generally considered to prolong the time before a total knee replacement, otherwise known as total knee arthroplasty (TKA), is required. During knee replacement surgery, the bone cuts and ligament balancing are 
done in such a way as to produce a straightened, mechanically improved lower extremity. The challenge for surgeons in the operating room is to accurately and efficiently evaluate the mechanical axis of the lower extremity. The medial mechanical axis deviation (MAD), in which the mechanical axis of the lower extremity lies more than $10 \mathrm{~mm}$ medial to the knee joint center, is considered a failed procedure [1. MAD is measured as the distance from the knee joint center to the line connecting the joint center of the hip and ankle (Figure 1(a)].

Recently, there has been increased interest in total knee arthroplasty with use of computer-assisted surgical (CAS) systems [2]3. These systems are designed to increase the precision of implantation of the components. The studies have found a clear tendency toward improved alignment of the limb and the component position with use of computer-assisted surgical navigation. Another study indicated that there is no significant difference between TKA with use of computer-assisted surgical navigation and conventional TKA using X-ray [3]. Most of the complications are surgery related and not due to computer navigation except for the cases of system down or software failure. Nevertheless, limitations far exceeded the positive attributes, which include a learning curve for surgeons using the CAS systems, hefty cost, a cumbersome system setup including line of sight for tracking and on-site calibration, and difficult system registration [23]. Apart from using navigation systems that determine the axis, the most popular method in clinical practice is electrocautery cord and X-ray grid (Figure 1(b)], consisting of reference lines, which were proposed to verify the mechanical axis alignment 45 . With this process, a large number of X-ray images and consequently, exposure, are required to achieve recommended MAD measures. All necessary data for calculating the axis model is collected during the operation. Of critical importance, the joint centers must be located at the center of the X-ray image. This enables the surgeon to mentally assess and estimate lower limb axis alignment. The leg bones are repositioned and several other X-ray images are re-acquired to reassess axis alignment. This iterative process is repeated until there is surgeon satisfaction with respect to MAD tolerance. A postoperative radiograph is then acquired to verify a positive outcome of the surgery. In order to alleviate radiation exposure from these conventional methods, authors in [6] considered MRI, however they claim a significantly underestimated MAD measurement.

We propose a clinical protocol that has the potential to offer robust intraoperative assessment of MAD to the surgeon. Our protocol requires that the X-ray images cover the femoral head, the knee and the ankle for MAD measurements. These anatomical landmarks do not require central location in the image. The registration of the three $\mathrm{X}$-ray images to the panorama is realized by an optical video camera attached to the $\mathrm{C}$-arm fluoroscope gantry viewing a visual marker pattern underneath the operating table [7]. Thanks to a one-time calibration between the camera and the X-ray source [8], our system allows for real-time, radiation-free $\mathrm{C}$-arm motion estimation. This low cost solution can be translated seamlessly in the current clinical setting and does not require additional hardware or calibration during surgery. The method has been systematically analyzed 


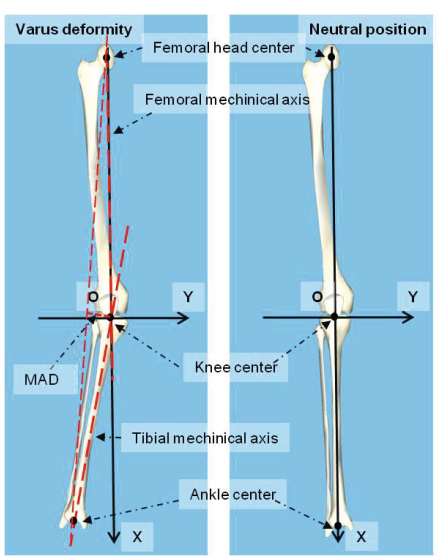

(a)

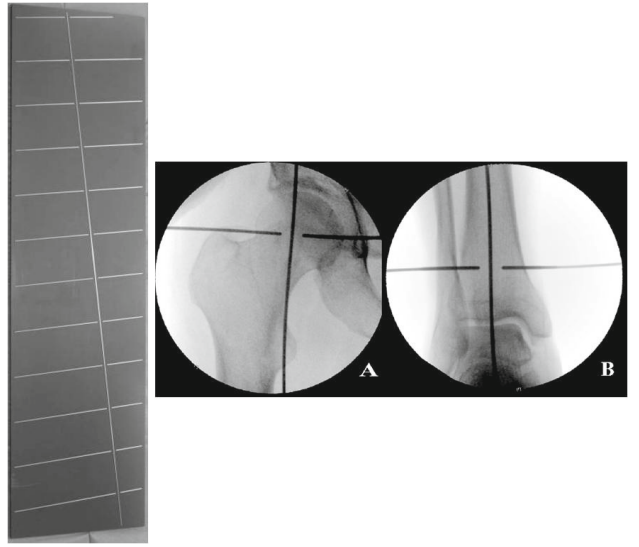

(b)

Fig. 1. (a) The mechanical axis deviation, MAD, is defined as the perpendicular distance between knee center and the line joining hip and ankle center. The left and right images show varus deformity and neutral position of the leg; (b) One standard in clinical practice is X-ray grid method for intraoperative axis alignment. The left image shows the X-ray grid board and the right image shows centres of the femoral head and of the ankle joint aligned with the reference lines of the board. Image courtesy of Liodakis et al. 9].

in a simulation framework in order to investigate its accuracy and robustness against potential error sources. we evaluate our new protocol by presenting results comparing the MAD measurements of 4 surgeons between ground-truth CT and X-ray panorama images obtained from 19 cadaver legs.

\section{Methodology}

\subsection{X-ray Panorama Using Only 3 Images}

The key step of image stitching is the estimation of the planar transformation for aligning images. Let a rotation $R_{i} \in \mathbb{R}^{3 \times 3}$ and a translation $t_{i} \in \mathbb{R}^{3}$ be defined from the coordinate system of the $i$-th camera view to the first camera view. In [10], the planar homography that aligns the $i$-th camera image to the first camera image is defined by

$$
H_{i}=K R_{i} K^{-1}+\frac{1}{d_{i}} K t_{i} n_{i}^{T} K^{-1}
$$

where $K \in \mathbb{R}^{3 \times 3}$ is the intrinsic matrix of the camera. $H_{i}$ is valid for all image points whose corresponding space points are on the same plane, i.e., stitching plane, defined by the normal vector $n_{i} \in \mathbb{R}^{3}$ and distance $d_{i}$ to the origin in the coordinate system of the $i$-th camera view.

Metric measurements are possible for the 3D space plane whose plane parameters in the camera coordinate system are known [7]. The coordinate of a 
$3 \mathrm{D}$ space point $P \in \mathbb{R}^{3}$ in the first camera view can be derived from its image projection $m_{i} \in \mathbb{R}^{3}$ (expressed in homogeneous coordinates) of the $i$-th camera view by,

$$
P=\frac{d_{1} K^{-1}\left(K R_{i} K^{-1}+\frac{1}{t_{i}^{T} n_{1}+d_{1}} K t_{i}\left(R_{i} n_{1}\right)^{T} K^{-1}\right) m_{i}}{n_{1}^{T} K^{-1}\left(K R_{i} K^{-1}+\frac{1}{t_{i}{ }^{T} n_{1}+d_{1}} K t_{i}\left(R_{i} n_{1}\right)^{T} K^{-1}\right) m_{i}}
$$

With the estimated coordinates of the 3D points, the metric measurements between the points can be calculated.

\subsection{The Limb Model Using Frontal Parallel Setup}

We define the coordinate systems for the limb and C-arm, and then specify the frontal parallel setup for the limb and the C-arm. The limb coordinate system is first defined and its origin is at the knee center (see Figure 1(a). Z-axis is the neutral AP vector for the front knee. The femoral mechanical axis is aligned with the $\mathrm{X}$ axis. Let the knee frontal plane (i.e. bone plane) be the XY plane. In the limb coordinate system, the knee center and femoral head center are fixed, while the ankle center locates differently on the XY plane depending on the hip-knee-ankle (HKA) angle. The coordinate system of the C-arm X-ray is expressed so that the origin is at the $\mathrm{X}$-ray source center, the $\mathrm{Z}$ axis is pointing and perpendicular to the detector, and $\mathrm{X}$ and $\mathrm{Y}$ axes are along the image width and height (see Figure 2). Frontal parallel setup, i.e. the bone plane is parallel to the C-arm detector plane, defines the norm as $\left[\begin{array}{lll}0 & 0 & 1\end{array}\right]^{T}$ and distance as the translation along the $\mathrm{Z}$ axis of the $\mathrm{C}$-arm. This setup is a commonly required clinical setup for enabling metric measurement in evaluating the frontal plane knee alignment [45] and X-ray image stitching [710]. It is usually verified by one control X-ray image showing the patella facing upwards [4/5].

\subsection{Simulation Study}

In the simulation study, let the $\mathrm{C}$-arm coordinate system of acquiring the control $\mathrm{X}$-ray image be the world coordinate system, whereby the control X-ray image defines the reference panorama frame. We set femur length (from the femoral head center to the knee center) and tibia length (from the knee center to the ankle center) to be $421 \mathrm{~mm}$ and $355 \mathrm{~mm}$. We set HKA to be $5^{\circ}$ and the true value of the corresponding MAD is accordingly $16.8 \mathrm{~mm}$. The limb is positioned relative to the first $\mathrm{C}$-arm view (the world coordinate system), such that it satisfies the frontal parallel setup. A frontal parallel setup constrains the norm of the frontal knee plane, i.e. no rotation around $\mathrm{X}$ and $\mathrm{Y}$ axes in the world coordinate system. In the frontal parallel setup, the distance of the bone plane to the origin is the translation along $\mathrm{Z}$ axis in the world coordinate system. The distance is unknown and can be arbitrary. General C-arm open space between the X-ray gantry and the detector is typically $1000 \mathrm{~mm}$, and the patient is positioned close to the center nearer to the $\mathrm{C}$-arm intensifier to achieve a larger field of view for 


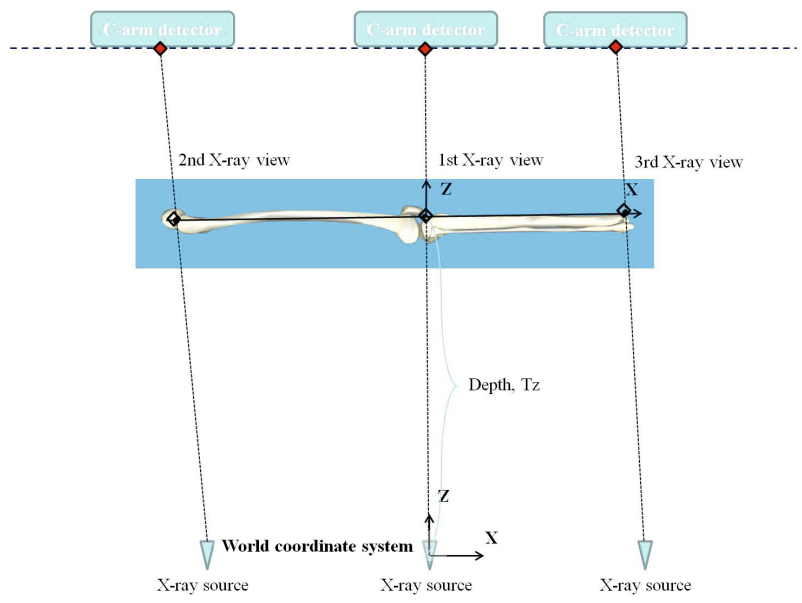

Fig. 2. Limb coordinate system definition. Its origin is at the knee center. $\mathrm{Z}$ axis is the neutral AP vector for the front knee. The femoral mechanical axis is aligned with the $\mathrm{X}$ axis. The knee frontal plane (i.e. bone plane) is defined be the XY plane.

the surgeon. For this reason, we choose $700 \mathrm{~mm}$ for the distance. We initially set rotations and translation about XYZ to be zero. The above parameter settings fix the transformation from the limb to the world coordinate system, which defines the frontal parallel setup used in our simulation. Measurement errors are always a possibility during a typical surgery primarily due to the distance of the bone plane not being accurately calculated or not being in the frontal parallel setup. To correct for these [7] require a manual estimation of the distance between the bone plane and marker pattern plane. Alternatively, 10 proposed to align the contours of the bone plane in two X-ray images for recovering the measurement error caused by the incorrect distance. We choose the former option as our solution does not require the overlapping area between the X-ray images in order to further reduce radiation exposure.

For clarity, we use the term estimated setup to represent the frontal parallel setup, since it is not the true actual setup. We make estimated setup constant in our simulation study, since most clinical methods for panorama generation and measurements are based on the assumption of frontal parallel setup. The actual pose of the limb is obtained by adding deviations to the estimated setup. To simulate the image projections of the three anatomical points in the individual images and the C-arm motions between image acquisitions, the actual pose of the limb is applied. To stitch the images (i.e. computing the coordinates of the imaged points in the panorama frame) and perform the measurements, we use the estimated pose of the limb. For each of following simulation experiments, we perform 1000 trails to compute the difference between the estimated and true MAD values as the final MAD errors. 


\subsection{Preclinical Study}

19 human cadaver legs were used and manually bent to introduce a random valgus or varus misalignment while keeping the knee fully extended. Each individual leg was positioned in a wooden box and placed on a carbon table with a visual marker pattern of $2378 \times 1682 \mathrm{~mm}$ rigidly attached underneath it. The leg positioning was such that the patella faced upwards (i.e. confirmed by an X-ray image). The Siemens Powermobile C-arm (Erlangen, Germany) was translated along the carbon table in a PA gantry position for acquiring the three X-ray images. Three X-ray images of femoral head, the knee and the ankle were acquired and subsequently stitched together. A CT scan of the leg was conducted immediately after (i) to ensure that we have the same leg position between X-ray stitching and CT, and (ii) as ground truth reference comparison. Four clinicians with different experience levels (i.e. 2 expert surgeons, 1 resident surgeon, and 1 last year medical student) were involved in our study. The anatomical landmarks defining the mechanical axis and the knee center were manually determined by each observer individually for all of the CT images and panoramic X-ray images in order to allow MAD calculation. For the CT measurement we used the K-Pacs Software v.1.6.0.

\section{Results and Discussion}

A $10 \mathrm{~mm}$ MAD value is a typical range of the healthy patients 1]. In clinical practice, a successful operation of lower limb correction is confirmed by a $0 \mathrm{~mm}$ of MAD after operation. Therefore, we use $10 \mathrm{~mm}$ as the MAD error tolerance to ensure the actual value of MAD is less than $10 \mathrm{~mm}$ when its measured value is $0 \mathrm{~mm}$.

Simulation Study Results: We simulate the inaccurate frontal parallel setup by adding random errors within $\pm 5^{\circ}$ to the rotations around XYZ axes of the limb pose. The mean MAD error calculated is: $0.52 \pm 0.51 \mathrm{~mm}$. The image coordinates of the anatomical points are manually selected by surgeons in clinical practice. The incorrect determination of the image coordinates, depending on experience of the surgeon, is simulated by adding random errors within \pm 10 pixels to the true image coordinates of hip-knee-ankle. The mean MAD error calculated is: $1.56 \pm 1.07 \mathrm{~mm}$. To assess the influence of the inaccurate distance of the bone plane on the MAD measurements, we add distance errors to ruler readings between $-100 \mathrm{~mm}$ to $100 \mathrm{~mm}$ with the increments of $20 \mathrm{~mm}$ to the true distance. For each distance error, we compute MAD errors using 1000 trails with and without applying random errors of the rotations and image coordinates. Figure 3 shows the results and indicates that it is important to accurately estimate distance of bone to source.

Preclinical Results: CT-based measurement is regarded as gold-standard. The change in measured MAD values between $\mathrm{CT}$ and the proposed method is the MAD error. First a t-test was performed; using CT data MAD values of $14.75 \pm$ $9.16 \mathrm{~mm}$ were achieved compared to $13.25 \pm 7.66 \mathrm{~mm}$ when using the $\mathrm{C}$-arm 

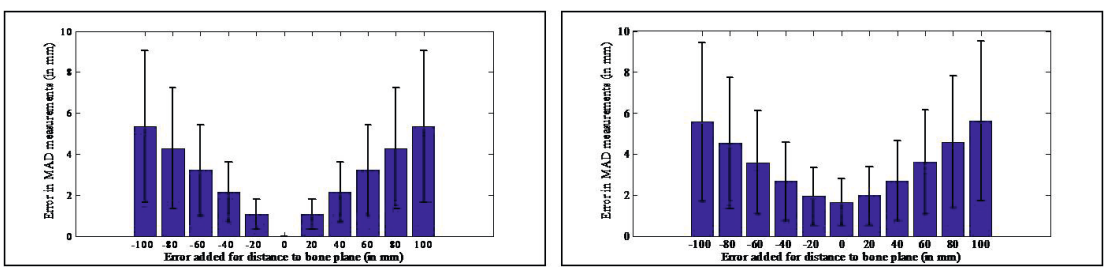

Fig. 3. Average and standard deviation MAD errors, in millimeters, for (Left), distance errors on ruler reading alone, and (Right) both distance errors on rulers coupled with pixel errors in the $\mathrm{X}$-ray images

stitching data. From this, there is no significant difference in the measured data $(p>0.1)$. Secondly, we performed a Pearson Correlation that displayed a highly significant correlation between CT and our method $(r=0.96)$. We performed an error analysis of 100 trials for each of the 19 cadaver legs by calculating the mean and standard deviations of mechanical axis deviation when adding random errors described in Figure 4 . Results for each participant compared to groundtruth CT show that the MAD error is well below the recommendation of $10 \mathrm{~mm}$ 1] for HTO and TKA procedures guaranteeing axis alignment. The MAD errors between all the examiners are below $1 \mathrm{~mm}$. The student is slightly better. This is another proof that the proposed method is reliable against inter-observers. Our maximum 3.5mm MAD error shows our method robustness. Note that Leg alignment involves multiple MAD measurements. For the entire procedure, the effect of our proposed MAD measurement method will be multiplied. As agreed upon by our three expert surgeons, an average of 8-12 X-ray images are typically acquired for one MAD measurement compared to our 3.

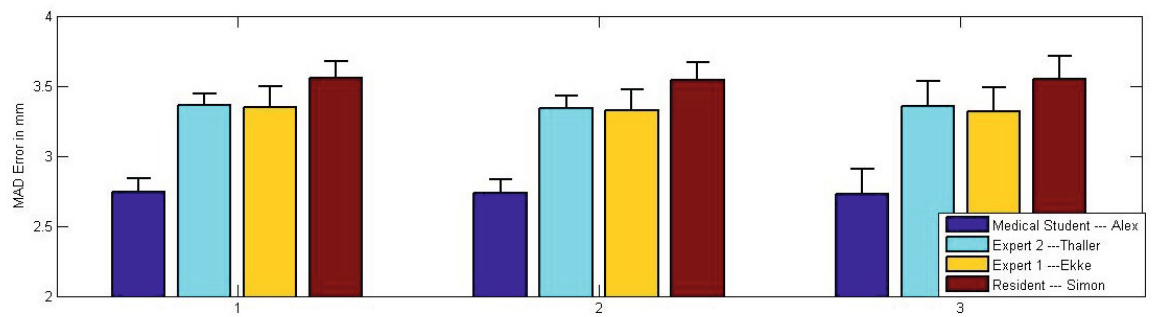

Fig. 4. An average MAD error of at most $3.5 \mathrm{~mm}$ for the mechanical axis deviation compared to ground-truth CT shows the robustness of our protocol. 1- errors only added to hip-ankle-knee pixel coordinates in X-ray; 2- errors added to ruler height; 3the combination of both errors in X-ray and ruler.

\section{Conclusion}

Existing methods and solutions for MAD intraoperative calculation demand a high level of surgeon experience since both hip and ankle centers have to be 
brought on a common grid or reference line and be located in the center of the $\mathrm{X}$-ray [45. This results in a complex procedure and many X-ray shots. Our proposed technique requires only three $\mathrm{X}$-ray images to be acquired showing the hip center, the knee center and the ankle center, thereby reducing procedural time. Via a one-time ruler measurement of the distance between marker pattern and knee, we always achieve accurate intraoperative MAD measurements enabling our protocol to become a potential alternative to standard clinical practice. Furthermore, our protocol can be generalized to any standard C-arm worldwide as long as the C-arm pose is available. We await ethics board approval for a patient study evaluation.

\section{References}

1. Paley, D.: Principles of Deformity Correction, 2003 edn. Springer (April 2003)

2. Kim, Y., Kim, J., Yoon, S.: Alignment and orientation of the components in total knee replacement with and without navigation support: a prospective, randomised study. The Journal of Bone and Joint Surgery 89(4), 471-476 (2007)

3. Kim, Y., Kim, J., Choi, Y., Kwon, O.: Computer-assisted surgical navigation does not improve the alignment and orientation of the components in total knee arthroplasty. The Journal of Bone and Joint Surgery 91(1), 14-19 (2009)

4. Saleh, M., Harriman, P., Edwards, D.J.: A radiological method for producing precise limb alignment. The Journal of Bone and Joint Surgery 73(3), 515-516 (1991)

5. Krettek, C., Miclau, T., Grn, O., Schandelmaier, P., Tscherne, H.: Intraoperative control of axes, rotation and length in femoral and tibial fractures. Technical Note 29(suppl.3), 29-39 (1998)

6. Liodakis, E., Kenawey, M., Doxastaki, I., Krettek, C., Haasper, C., Hankemeier, S.: Upright MRI measurement of mechanical axis and frontal plane alignment as a new technique: a comparative study with weight bearing full length radiographs. Skeletal Radiology 40(7), 885-889 (2011)

7. Wang, L., Traub, J., Weidert, S., Heining, S.M., Euler, E., Navab, N.: Parallax-free intra-operative x-ray image stitching. Med. Imag. Anal. 14(5), 674-686 (2010)

8. Navab, N., Heining, S.M., Traub, J.: Camera augmented mobile c-arm (camc): Calibration, accuracy study and clinical applications. IEEE Trans. Med. Imag. 29, 1412-1423 (2010)

9. Liodakis, E., Kenawey, M., Liodaki, E., Mommsen, P., Krettek, C., Hankemeier, S.: The axis-board: An alternative to the cable technique for intraoperative assessment of lower limb alignment. Technology and Health Care 18(3), 165-171 (2010)

10. Yaniv, Z., Joskowicz, L.: Long bone panoramas from fluoroscopic x-ray images. IEEE Transactions on Medical Imaging 23(1), 26-35 (2004) 